American Journal of Pharmaceutical Education 2019; 83 (5) Article 6781.

\title{
RESEARCH
}

\section{The Positive Impact of an Extended Intervention on Dosing Accuracy of Student Compounded Suspensions}

\author{
Emily C. Darst, PharmD, ${ }^{a}$ Robert P. Shrewsbury, $\mathrm{PhD}^{\mathrm{b}}$ \\ ${ }^{a}$ College of Pharmacy and Pharmaceutical Sciences, Washington State University, Spokane, Washington \\ ${ }^{b}$ Eshelman School of Pharmacy, University of North Carolina, Chapel Hill, North Carolina
}

Submitted September 7, 2017; accepted January 5, 2018; published June 2019.

\begin{abstract}
Objective. To demonstrate the impact of an extended intervention on the dose accuracy and consistency of a compounded zonisamide suspension.

Methods. A laboratory exercise was initially conducted by pharmacy students to determine the beyond-use date (BUD) of a compounded zonisamide suspension. The student results were inconsistent with data in a published reference study. The exercise was repeated several times testing various hypotheses to explain the inconsistency. The final hypothesis was the student techniques of shaking and sampling their suspensions resulted in inaccurate samples. Therefore, a final hypothesis study was designed to include an extended intervention (weeks 5-7) that would demonstrate the impact of explicit verbal and visual instructions on the proper shaking and sampling of suspensions on dose accuracy and consistency.

Results. The initial study found that students' weekly average zonisamide potencies ranged from 71\%$122 \%$ of label, with a relative standard deviation (RSD) of $17 \%-53 \%$; weekly potencies in the reference study had ranged from $92 \%-105 \%$. In the final hypothesis study before the extended intervention, dosing accuracy ranged from 64\%-111\% (RSD 17\%-76\%). During the 3 week long intervention, dosing accuracy became $91 \%-118 \%$ with a RSD of 5\%-29\% which were consistent with the reference study.

Conclusion. Providing more explicit auditory and visual instructions to pharmacy students regarding the proper shaking and sampling techniques of their compounded suspensions resulted in more consistent and accurate dosing of a zonisamide suspension. By implication, pharmacists providing specific and personalized instructions to patients should reduce their self-dosing inconsistencies at home.
\end{abstract}

Keywords: suspensions, improper dosing, intervention study, compounding, shaking

\section{INTRODUCTION}

Maximum therapeutic outcomes are achieved when the proper dose of the correct drug is given to a patient at the optimum dosing time. One of the goals of pharmacy counseling is to help patients know and understand these variables. Pharmacist counseling is particularly important when patients have to prepare doses of a suspension at home since the accuracy of those doses will have a large impact on the patients' therapeutic outcomes. The manner in which this pharmacy counseling takes place is also an important variable in the patients' response to the instructions and information.

Corresponding Author: Robert P. Shrewsbury, Eshelman School of Pharmacy, University of North Carolina at Chapel Hill, 301 Pharmacy Ln., Chapel Hill, NC 27599-7574. Tel: 919-962-0093. E-mail: bob_shrewsbury@unc.edu
Suspensions require proper and adequate shaking to produce a uniform preparation prior to each dose administration since dispersed particles have the potential to settle and develop floccules between doses. Therefore, suspensions require more effort on the part of a patient at the time of dosing than do capsules, for example, which are already in unit doses. The proper dosing of suspensions relies on the patient's understanding and ability to carry out instructions for preparing correct doses. The responsibility for imparting this education to the patient rests with the pharmacist at the time a suspension is dispensed. Therefore, important goals in pharmacy education in addition to preparing correctly compounded preparations are to train pharmacy students to know how to properly prepare and administer doses from the preparations and successfully impart this knowledge to patients. 


\section{American Journal of Pharmaceutical Education 2019; 83 (5) Article 6781.}

One of the laboratory exercises carried out at the University of North Carolina Eshelman School of Pharmacy was designed to teach students the process by which beyond-use dates (BUDs) are determined for compounded suspensions. The students compounded a zonisamide suspension, withdrew samples from their suspension once a week for several weeks, and used the analytical results to calculate the BUD for the suspension. The laboratory exercise was modeled after a published research study that followed a very common compounding scenario: use capsules as the source of the active pharmaceutical ingredient (API) and prepare a suspension using either a commercially available vehicle or a vehicle that was itself compounded. ${ }^{1}$ According to the published study, zonisamide remained stable for at least 28 days in a vehicle of simple syrup or $0.5 \%$ methylcellulose at both refrigerated and room temperatures. However, when the students completed a similar exercise, their data indicated that zonisamide was not stable in either suspension vehicle, regardless of the storage temperature. This report details the investigative path used to determine why the initial students' data differed from the published reference. The entire investigation also demonstrates the necessity and utility of having analytical data for student compounded preparations.

\section{METHODS}

Second-year pharmacy (P2) students followed the methods described in the Formulation Record to prepare a zonisamide suspension (Appendix 1). The students drew samples from their own preparation every week for the duration of the study. The students received the following verbal instructions before obtaining their weekly samples: "Provide a $0.5 \mathrm{~mL}$ sample as if you are giving a patient a dose." Using an oral syringe, the students collected the $0.5 \mathrm{~mL}$ sample from their prescription bottle, which was fitted with an oral syringe adapter. The sample was placed in a scintillation vial, labeled with a unique identification code, and left for the compounding staff to analyze using high performance liquid chromatography (HPLC).

The compounding staff completed the analysis each week within a few hours after the students had collected their samples. The sample work-up consisted of adding $19.5 \mathrm{~mL}$ of mobile phase with $0.5 \mathrm{mg} / \mathrm{mL}$ internal standard (methylparaben) to the $0.5 \mathrm{~mL}$ sample in each scintillation vial. The vial was placed in a vortex mixer for 10 seconds and left to stand for 5 minutes. A $1 \mathrm{~mL}$ sample of the mixed solution was placed in a new scintillation vial, and $9.0 \mathrm{~mL}$ of mobile phase (without internal standard) was added. The vial was shaken by hand for 10 seconds, and a volume of supernatant was removed for analysis.
The HPLC mobile phase was 3:7 acetonitrile and $20 \mathrm{mM}$ sodium monobasic phosphate. The $\mathrm{pH}$ of the mobile phase was adjusted to 3.5 using phosphoric acid. Twenty-five microliters from each sample was injected once using a Waters 717 Plus auto-injector (Waters Corporation, Milford MA) onto a 4.6 x $250 \mathrm{~mm} \mathrm{C18} 10 \mu \mathrm{L}$ Thermo Scientific (Thermo Fisher Scientific, Waltham MA) column with a mobile phase flow rate of 1.4 $\mathrm{mL} / \mathrm{min}$. The system is designed to back-flush through the injection tubing between each sample. This ensured all residual liquid from the previous sample was removed from the system before the next injection. A Dionex U1timate 3000 UV/VIS detector (Thermo Fisher Scientific, Waltham MA) was set at a wavelength of $\lambda=240$. The retention times of zonisamide and methylparaben were 4.8 and 6.0 minutes, respectively. Data collection was processed using the Chromeleon 6.8 SR11 chromatography data system (Thermo Fisher Scientific, Waltham MA), and Excel (Microsoft, Redmond, WA) was used to process computations and produce spreadsheets and graphics. The results for all samples from each compounded suspension were calculated as the percent of label of zonisamide. Duplicate samples and multiple injections were not done because of the very large number of samples and the need for a rapid turnaround time of the data.

This initial study data showed the students' results were inconsistent with the findings of the published reference study. This led the instructors to identify several potential causes. One possible though unlikely cause was that the data from the literature reference itself was flawed and failed to show zonisamide degradation. However, a greater concern was that only one set of standards had been prepared on day 0 and those values had been used to represent $100 \%$ of label for the entire study. Therefore, all of the students' samples for each week had been calculated from this day 0 "percent of label" standard value. Another possibility could have been that this was the first time such an exercise was conducted in this laboratory, so its reproducibility or lack thereof needed to be verified by repeating the study. There was also the potential that the HPLC analysis procedures or the instrumentation were responsible for the widely varying results.

The group of students who completed the initial study could not repeat it due to curricular time constraints. Instead, the same exercise was repeated three times during subsequent years with different groups of students using the same compounding and sample collection scheme, analytical process, and instrumentation. Each time, the student data was inconsistent with the published study. Because different groups of students were involved in each year's study, the possibility that only one group of 


\section{American Journal of Pharmaceutical Education 2019; 83 (5) Article 6781.}

students had been responsible for creating the observed data inconsistency was dismissed.

The process of computing student data using only one set of standard values seemed to be unlikely because the standards values across the different iterations were found to be very similar. In addition, HPLC parameters such as RSD of the internal standard AUC and the theoretical plates of the HPLC column differed by less than $2 \%$ between the studies, suggesting that the analytical procedures were also not responsible for the inconsistencies. All of these results strongly indicated that a systemic problem existed in either the study design or the study execution.

Therefore, it was finally hypothesized that the inconsistent results most likely came from the process by which students were shaking the suspension and drawing their weekly samples. This unforeseen error was not considered when the study design was first conducted since the students were not explicitly instructed in or shown how to shake a suspension prescription bottle, nor was their sampling technique observed. Therefore, the objective of the final hypothesis study was to envision that each collected sample was a "dose" in a patient's therapeutic regimen.

To quickly and informally test this hypothesis, a short study was performed by the course instructor and teaching assistants $(n=6)$ who were explicitly instructed on proper shaking technique and syringe use prior to and repeatedly throughout the trial period. Each participant prepared all four types of suspensions in the $2 \times 2$ matrix described in the published study, resulting in 24 preparations. The percent of label results were consistent with the reference study showing that zonisamide did not degrade over the 16-day period. These results supported the conclusion that this final hypothesis was the reason for the inconsistencies between the students' findings and the published study.

The "final hypothesis study" was conducted in spring 2016 and designed to start in the same manner as all of the other studies, i.e., without giving students explicit instructions of how to shake the suspension prescription bottle or observing how they collected their samples. It was anticipated that the data from this group of students in this 4-week pre-intervention segment (weeks 1-4) would resemble the data from all of the other studies. Then to demonstrate that the techniques of shaking and drawing a sample were the true cause of the inconsistent results, weeks 5-7 (post-intervention) were conducted with instructors and teaching assistants repeatedly giving specific verbal instructions and demonstrations to students. Students were to shake the prescription bottle vigorously, observe the bottom of the bottle for any remaining sediment not yet suspended, and examine the uniformity of the suspension. The sample was to be drawn immediately after shaking the bottle, ensuring no air bubbles were present in the syringe, and withdrawing the correct volume. Students were not allowed to draw their sample until an instructor or teaching assistant visually monitored the shaking of their suspension and their sampling procedure. The activities of the instructors and the teaching assistants served as the intervention for the students' technique, and represented a pharmacist-initiated patient education activity. The analysis of the samples collected represented the "doses" a patient was about to administer.

A strategy for standards in this final study was also different than in previous studies. A new standard suspension of each suspension type in the $2 \times 2$ matrix was compounded every week that students drew and submitted samples. The standards were also analyzed week after week and compared with the newly prepared standards to make certain that the standard preparations were in fact not degrading over time. The standard preparations were compounded and sampled by the same laboratory staff member for the entire duration of the study to ensure uniformity of technique. The one exception was the room temperature (RT) samples on day 0 . Because those samples were drawn by a different person and resulted in values clearly inconsistent with all of the other standards data, the standard value used in the calculation of the student sample analyses on day 0 was the day 7 standard for the corresponding preparation/temperature. Analyses of all standard samples were conducted at the same time as the corresponding student samples. The calculated analysis values for the standards were the AUC ratio of zonisamide/internal standard from the HPLC assay. As with the student samples, only one injection of each standard was analyzed.

\section{RESULTS}

Table 1 displays the averaged zonisamide potency for each subgroup in the initial study and the data from the literature reference. Some individual student results varied with a potency of $1 \%$ to over $200 \%$ of label. There were no day 7 data in the initial study because spring break occurred that week and the students were not present to sample their compounded suspensions.

There were 131 students in the final hypothesis study (Table 2). Eighty-seven (87) students were included in two Friday laboratory sections, and 43 students were included in the one Wednesday laboratory section. Unfortunately, inclement weather caused the university to close which disrupted the coordination of the groups within the study. As a result, only 87 students' data (the Friday group) had a time-series analysis result similar to the initial study. 


\section{American Journal of Pharmaceutical Education 2019; 83 (5) Article 6781.}

Table 1. Zonisamide Potency in the Initial Study and the Literature Reference Study (Mean (Standard Deviation) [Relative Standard Deviation])

\begin{tabular}{|c|c|c|c|c|}
\hline \multirow[b]{2}{*}{ Day } & \multicolumn{2}{|c|}{ Refrigeration } & \multicolumn{2}{|c|}{ Room Temperature } \\
\hline & SS & MC & SS & MC \\
\hline 0 & $95.6(27.5)[28]$ & $105.8(45.7)[43]$ & $100.1(21.1)[21]$ & $99.3(25.9)[26$ \\
\hline 14 & $93.9(35.8)[38]$ & 96.9 (39.5) [41] & $91.6(29.8)$ [33] & $108.2(25.1)[23]$ \\
\hline 21 & $86.5(37.8)[44]$ & $93.1(38.5)[42]$ & $98.1(26.7)[27]$ & $123.0(21.5)[17]$ \\
\hline 28 & $71.5(35.4)[49]$ & $79.6(41.6)$ [53] & $87.9(26.2)[30]$ & $103.4(18.4)[17]$ \\
\hline \multicolumn{5}{|c|}{ Literature Reference Study ${ }^{1}$} \\
\hline 0 & $102(18)[18]$ & 99 (6) [6] & 99 (2) [2] & 99 (9) [9] \\
\hline 14 & 100 (4) [4] & $92(6)[7]$ & 98 (15) [15] & 102 (1) [1] \\
\hline 21 & 101 (14) [14] & $101(3)[3]$ & 105 (9) [9] & $92(15)[16]$ \\
\hline 28 & 95 (19) [20] & $104(7)[7]$ & 102 (11) [11] & 103 (6) [6] \\
\hline
\end{tabular}

Abbreviations: $\mathrm{SS}=$ simple syrup, $\mathrm{MC}=$ methylcellulose, $\mathrm{Ref}=$ refrigeration, $\mathrm{RT}=$ room temperature

\# of replicates: SS Ref $n=34$, MC Ref $n=37$, SS RT $n=36$, MC RT $n=35$

Two comparisons were conducted using the RSD as a measure of variation in the students' performance. The first was between the initial study (Table 1) and the final hypothesis study pre-intervention data (Table 2) and the second was between the preparation type and storage temperature data for the initial study, the three iterative studies, and the pre-intervention final hypothesis study data (Table 3). In both of these comparisons, one-way ANOVAs consistently indicated a statistical difference between the studies regardless of the preparation type and storage temperature. Subsequent statistical comparison of groups using the Wald z-test identified the different studies, preparations, and storage temperatures that had a significant difference. However, there was no discernable pattern of error in either of these two comparisons.

Another statistical comparison strategy was to test for significant differences in parameters that would describe the time-course of the data throughout the studies (Table 4). Because any degradation of zonisamide in suspension was considered a first-order process, fitting the data to an exponential equation to generate an intercept and a slope parameter for each preparation/temperature data set was indicated. One-way ANOVAs consistently indicated a statistical difference between the parameters regardless of the preparation type and storage temperatures and subsequent Wald z-tests did identify significant differences between some groups. However, there was no consistent pattern of error.

The means, SDs, and RSDs of the analysis results during the post-intervention weeks in the final hypothesis study (Table 2) were consistently provided to the students as a method to demonstrate the impact of the ongoing intervention. A comparison of these data with the initial study data found smaller SDs and RSDs were obtained in the post-intervention data. The percent of label values also began to trend back to $100 \%$ for each preparation type and storage temperature as shown in Table 2 and Figure 1.

Figure 2 includes the data for all 131 students in the final hypothesis study. Combining all of the data allowed more days to be included in the pre-intervention portion of

Table 2. Zonisamide Potency in the Final Hypothesis Study (Mean (Standard Deviation) [Relative Standard Deviation])

\begin{tabular}{|c|c|c|c|c|}
\hline \multirow[b]{2}{*}{ Day } & \multicolumn{2}{|c|}{ Refrigeration } & \multicolumn{2}{|c|}{ Room Temperature } \\
\hline & SS & MC & SS & MC \\
\hline 0 & $98.8(17.5)[18]$ & $83.4(27,7)[33]$ & $75.4(34.3)[45]$ & $64.8(30.2)[47]$ \\
\hline 5 & $107.0(19.4)[18]$ & $81.3(34.8)[43]$ & $97.5(37.7)[39]$ & $111.2(18.8)[17]$ \\
\hline 14 & $104.3(37.6)[36]$ & $78.6(59.9)[76]$ & $72.4(31.8)[44]$ & $81.5(38.2)[47]$ \\
\hline 21 & $92.1(27.6)[30]$ & $84.2(33.5)[40]$ & 70.7 (42.0) [59] & $106.9(38.7)[36]$ \\
\hline \multicolumn{5}{|c|}{ Intervention } \\
\hline 28 & $112.3(6.2)[6]$ & $98.1(6.7)[7]$ & 96.5 (28.4) [29] & $91.6(14.6)[16]$ \\
\hline 35 & $118.0(6.8)[6]$ & $94.8(17.2)[18]$ & $101.0(22.2)[22]$ & $103.3(11.5)[11]$ \\
\hline 42 & $109.8(5.3)[5]$ & $98.9(7.9)[8]$ & $96.6(11.3)[12]$ & $102.2(13.3)[13]$ \\
\hline
\end{tabular}

Abbreviations: $\mathrm{SS}=$ simple syrup, $\mathrm{MC}=$ methylcellulose, $\mathrm{Ref}=$ refrigeration, $\mathrm{RT}=$ room temperature

\# of replicates: SS Ref $n=22$, MC Ref $n=21$, SS RT $n=22$, MC RT $n=22$ 


\section{American Journal of Pharmaceutical Education 2019; 83 (5) Article 6781.}

Table 3. Averaged Relative Standard Deviation in the Initial Study, Three Iterative Studies, and the Final Hypothesis Study

\begin{tabular}{|c|c|c|c|c|}
\hline & \multicolumn{2}{|c|}{ Refrigeration } & \multicolumn{2}{|c|}{$\begin{array}{c}\text { Room } \\
\text { Temperature }\end{array}$} \\
\hline & SS & MC & SS & MC \\
\hline Initial Study & 40 & 45 & 28 & 21 \\
\hline Iterative Study 1 & 25 & 26 & 16 & 21 \\
\hline Iterative Study 2 & 35 & 24 & 33 & 18 \\
\hline Iterative Study 3 & 34 & 23 & 17 & 26 \\
\hline $\begin{array}{l}\text { Final Hypothesis Study } \\
\text { (pre-intervention) }\end{array}$ & 26 & 48 & 47 & 37 \\
\hline $\begin{array}{l}\text { Final Hypothesis Study } \\
\text { (post-intervention) }\end{array}$ & 6 & 11 & 21 & 13 \\
\hline
\end{tabular}

Abbreviations: $\mathrm{SS}=$ simple syrup, $\mathrm{MC}=$ methylcellulose, $\mathrm{Ref}=$ refrigeration, $\mathrm{RT}=$ room temperature

\# of replicates: $\mathrm{n}=5$

the figure since the time-series data were different in the two laboratory sections. Visually, Figure 2 illustrates the same intervention effect as seen in Table 2 and Figure 1. Once the intervention was initiated, the zonisamide percent of label approached the expected $100 \%$, and the overall variation in the student data was substantially reduced.

The outcomes of the new standard strategy used in the final hypothesis study are shown in Tables 5 and 6 . Table 5 shows the results for each new standard that was compounded weekly. Table 6 shows the values of all standards that were followed throughout the course of the study. With the exception of data noted in the Methods section (the RT samples on day 0), the AUC ratio values are remarkably similar showing that there is no zonisamide degradation in either suspension vehicle.

\section{DISCUSSION}

The original objective of the laboratory exercise was to teach students the process of determining the BUD of a compounded suspension. When several iterations of the study yielded results inconsistent with those of the published study, it was suspected that the students' techniques of re-suspending and drawing samples were the source of the inaccurate findings. This hypothesis created an opportunity to teach pharmacy students the impact of a direct and specific pharmacist counseling intervention could have on a patient's ability to accurately self-dose a suspension.

Several hypotheses were investigated and dismissed before the final conclusion was reached that students' techniques of shaking and drawing samples were the reason for the inaccurate results. The first hypothesis was the initial study group had not done such a laboratory exercise before and therefore did not do the work correctly. When the study was repeated in other groups of pharmacy students, it was clear this hypothesis was incorrect. The RSD comparison between the initial study, three iterative studies, and the pre-intervention study showed there was large variability in the data regardless of the study and that a single source of the variability could not be identified. Thus, the data confirmed that students in the pre-intervention part of the final hypothesis study did not compound, shake, or sample their suspensions differently from students in the initial study and the three iterative studies.

Another hypothesis, though unlikely, was the published study had been incorrect showing that zonisamide did not degrade over time. This hypothesis was excluded because new standards made weekly and continuously analyzed throughout the study did not show zonisamide degradation (Tables 5 and 6). All of these samples were collected by the same person with the exception of the RT samples on day 0 and showed remarkable consistency from day to day. The strategy demonstrated that if person knew how to compound a suspension correctly multiple times and could sample correctly each time,

Table 4. Exponential Equation Parameters of Mean Potency Data from the Initial Study, Three Iterative Studies, and the Final Hypothesis Study (Pre-Intervention Data)

\begin{tabular}{|c|c|c|c|c|c|c|c|c|}
\hline & \multicolumn{4}{|c|}{ Refrigeration } & \multicolumn{4}{|c|}{ Room Temperature } \\
\hline & \multicolumn{2}{|c|}{ SS } & \multicolumn{2}{|c|}{ MC } & \multicolumn{2}{|c|}{ SS } & \multicolumn{2}{|c|}{ MC } \\
\hline & Intercept $^{\mathbf{a}}$ & Slope $^{\text {b }}$ & Intercept $^{\mathbf{a}}$ & Slope $^{\text {b }}$ & Intercept $^{\mathbf{a}}$ & Slope $^{\mathbf{b}}$ & Intercept $^{\mathbf{a}}$ & Slope $^{b}$ \\
\hline Initial Study & 100.18 & -0.0094 & 108.17 & -0.0093 & 99.79 & -0.0036 & 102.45 & 0.0034 \\
\hline Iterative Study 1 & 91.35 & -0.0057 & 100.40 & 0.0028 & 94.12 & -0.0017 & 100.87 & 0.0020 \\
\hline Iterative Study 2 & 105.24 & -0.00082 & 95.03 & 0.0024 & 96.15 & 0.0091 & 98.99 & -0.00039 \\
\hline Iterative Study 3 & 122.62 & -0.013 & 127.56 & -0.0077 & 144.38 & -0.0075 & 110.52 & -0.0071 \\
\hline $\begin{array}{l}\text { Definitive Study } \\
\text { (pre-intervention) }\end{array}$ & 104.07 & -0.0036 & 81.84 & -0.0000084 & 85.07 & -0.0082 & 77.23 & 0.014 \\
\hline
\end{tabular}

Abbreviations: $\mathrm{SS}=$ simple syrup, $\mathrm{MC}=$ methylcellulose, $\mathrm{Ref}=$ refrigeration, $\mathrm{RT}=$ room temperature

\# of replicates: $\mathrm{n}=5$

${ }^{a}$ percent of label

b $1 /$ days $^{-1}$ 
Final Hypothesis Study SS Ref

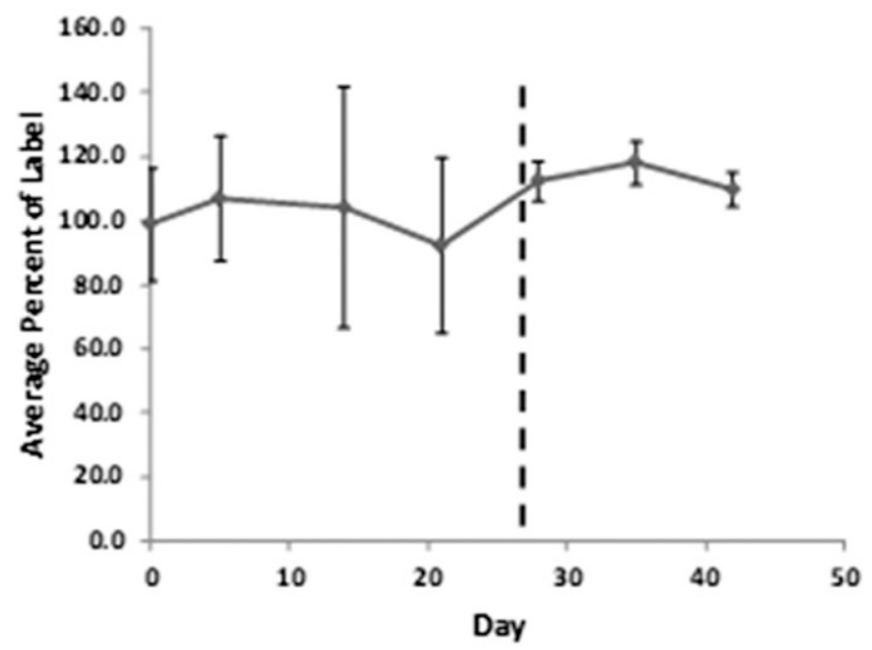

Final Hypothesis Study SS RT

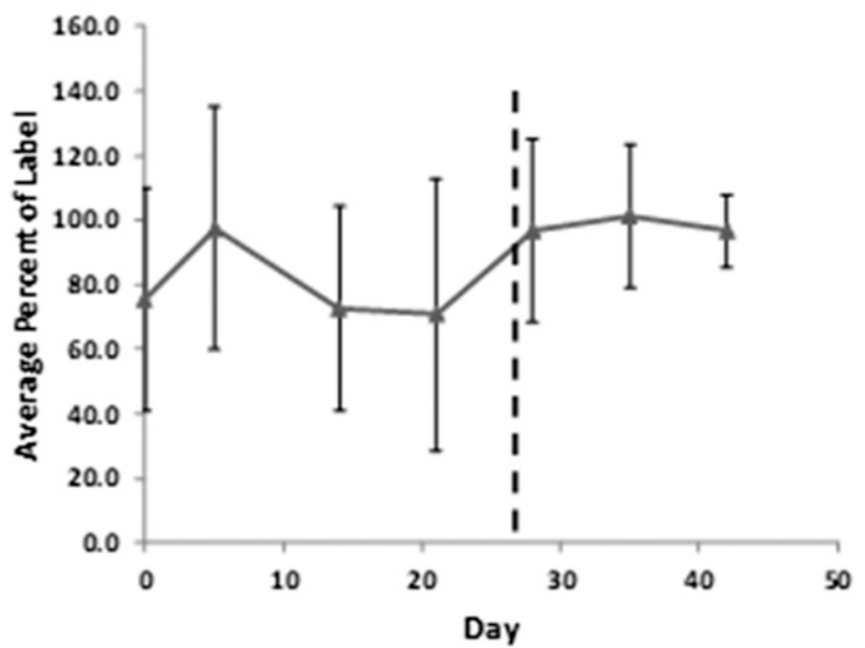

Final Hypothesis Study MC Ref

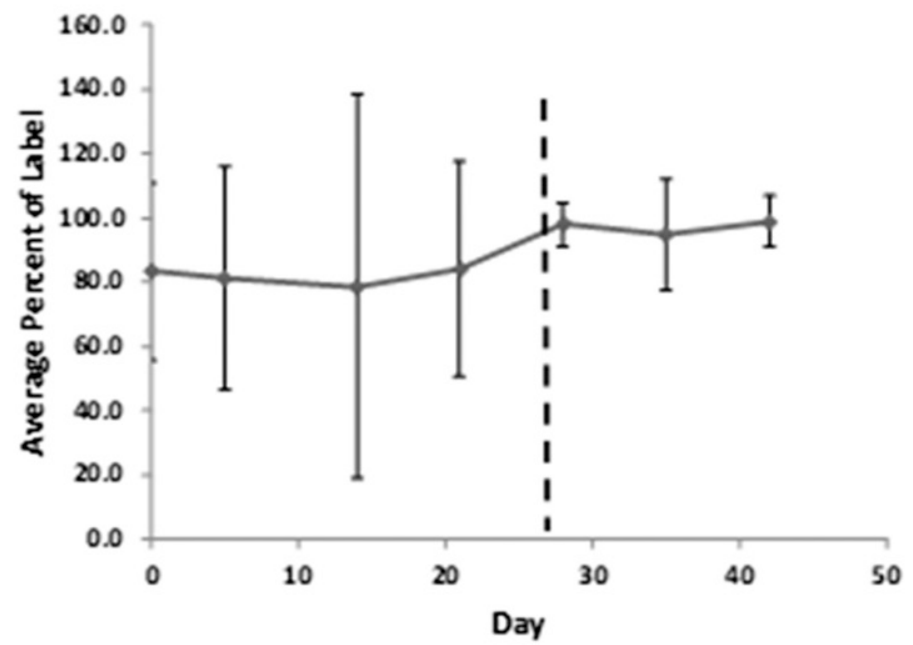

Final Hypothesis Study MC RT

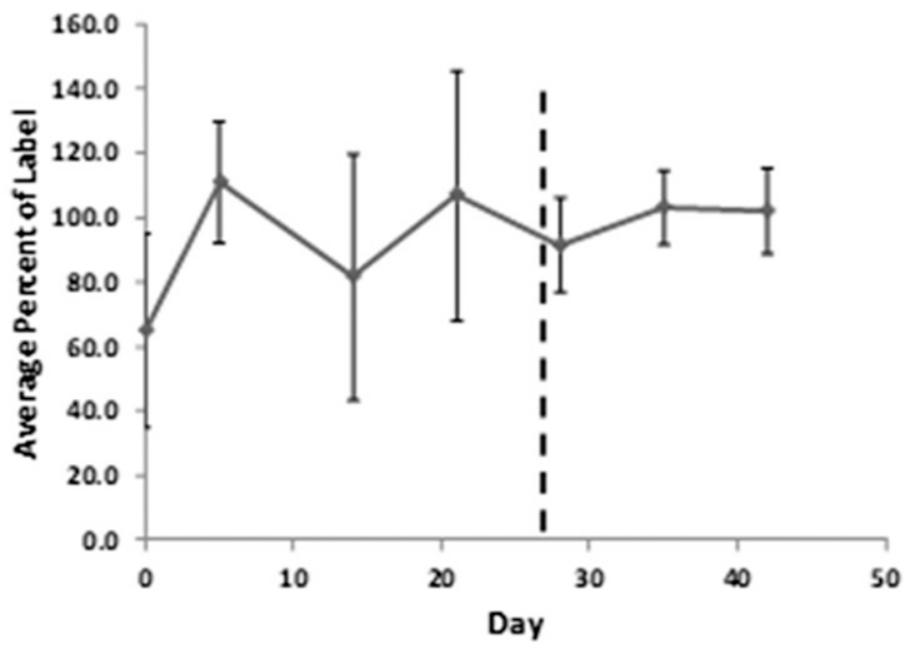

Figure 1. Mean Zonisamide Potency from the Final Hypothesis Study by Preparation Type and Storage Temperature (Dashed Line Shows Onset of Intervention)

Abbreviations: $\mathrm{SS}=$ simple syrup, $\mathrm{MC}=$ methylcellulose, $\mathrm{Ref}=$ refrigeration, $\mathrm{RT}=$ room temperature

\# of replicates: SS Ref $n=22$, MC Ref $n=21$, SS RT $n=22$, MC RT $n=22$

then consistent data can be obtained. The implication is that if a patient knew how to sample correctly multiple times, they would get consistent doses of their suspension which will provide optimum therapeutic outcomes. The standard strategy used in the final hypothesis study also validated the published study proving zonisamide does not degrade in these suspension vehicles over a period of 28 days. In our report, zonisamide suspensions did not degrade even after 42 days and potentially 49 days.

It was thought that zonisamide percent of label values in the initial study might have been incorrectly calculated because only one zonisamide standard com- pounded at the start of the study was used. The standard values in the three iterative studies suggested that this conclusion was unfounded. However, the definitive conclusion was again obtained from the standard strategy used in the final hypothesis study.

A potential central source of data variation in all of the studies was the high-pressure liquid chromatography (HPLC) analysis. It was fortunate that the same equipment was used and only the chromatographic column was different from study to study. All analysis parameters varied less than $2 \%$ from study to study indicating that the equipment and the column were performing at the same analytical specificity. 


\section{American Journal of Pharmaceutical Education 2019; 83 (5) Article 6781.}

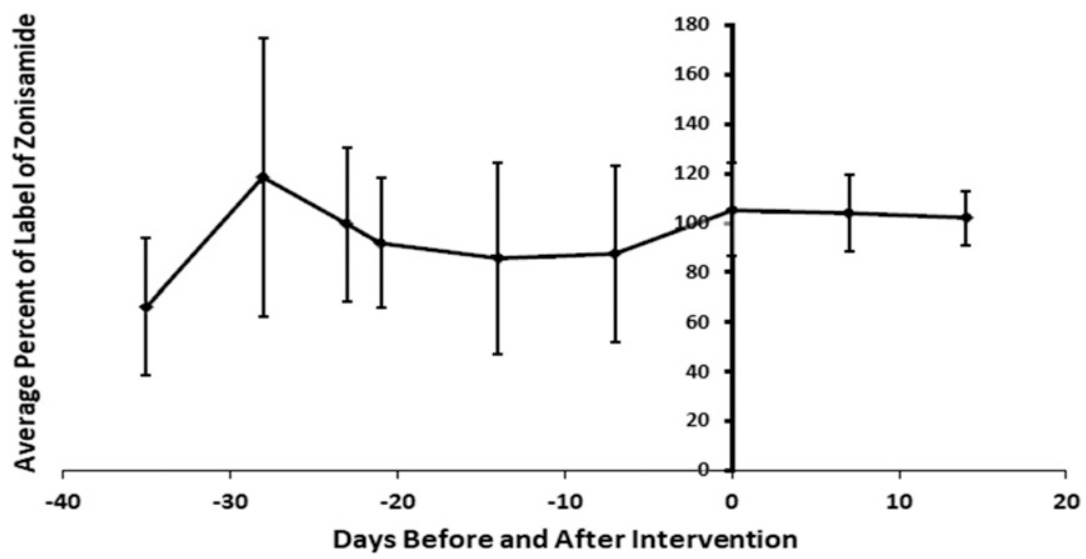

Figure 2. Mean Zonisamide Potency of All Students $(n=131)$ in the Final Hypothesis Study (Time 0 Denotes Start of Intervention)

With concern for the impact of counseling, the study demonstrated that simply providing instructions to "shake well" was not sufficient for students to properly and repeatedly obtain uniform doses. The findings of the study has led to a greater emphasis on teaching pharmacy students how to obtain an accurate dose from a suspension with the goal of becoming better equipped to teach their patients the same techniques. Also implied in the outcome of the study is instructions may need to be repeated. Therefore, detailed auditory and visual instructions regarding the proper shaking technique may need to be given to every patient each time a prescription is filled or refilled. Because a pharmacist cannot be present to shake liquid medications every time a patient needs a dose, it is imperative that pharmacists provide patients with sufficient and personalized counseling on how to correctly administer doses. This should include using specific verbiage geared toward the patient's level of understanding, demonstrating actions such as shaking, and asking the patient to repeat instructions and mirror the actions when necessary.

Suspensions are a unique dosage form since they require three specific "actions" on the part of the patient to ensure the correct dose is taken. Failure to complete any one of the action items correctly is an error in the dosing process. Ideally, pharmacists prefer not to require this much responsibility on the part of the patient due to the

Table 5. HPLC AUC Ratio of Zonisamide/Internal Standard for Each New Suspension Compounded Every Laboratory Period in the Final Hypothesis Study

\begin{tabular}{lcccccccc}
\hline Days & $\mathbf{0}$ & $\mathbf{7}$ & $\mathbf{1 4}$ & $\mathbf{2 1}$ & $\mathbf{2 8}$ & $\mathbf{3 5}$ & $\mathbf{4 2}$ & $\mathbf{4 9}$ \\
\hline SS RT & 0.33 & 0.54 & 0.69 & 0.72 & 0.71 & 0.65 & 0.70 & 0.68 \\
MC RT & 0.26 & 0.69 & 0.76 & 0.73 & 0.73 & 0.64 & 0.79 & 0.71 \\
SS Ref & 0.59 & 0.75 & 0.69 & 0.73 & 0.60 & 0.73 & 0.75 & - \\
MC Ref & 0.68 & 0.77 & 0.77 & 0.73 & 0.72 & 0.79 & 0.76 & - \\
\hline
\end{tabular}

Abbreviations: $\mathrm{SS}=$ simple syrup, $\mathrm{MC}=$ methylcellulose, $\mathrm{Ref}=$ refrigeration, $\mathrm{RT}=$ room temperature

\# of replicates: $\mathrm{n}=1$ potential of these large dosing errors. However, when a patient requires a liquid dosage form and the drug is insoluble in the vehicle, a suspension is the only viable option. Therefore, the patient is required to re-suspend the medication at home at the time of administration and properly shaking a suspension to ensure that dose uniformity is fundamental.

The study also points to the utility of pharmaceutical analysis as an assessment tool in student compounding laboratories. ${ }^{2-5}$ If the initial study had been conducted without sample analysis, the reason why students failed to mimic the literature study would have been unknown to the course instructor or if known, the instructor would have no resource to determine the cause. Pharmaceutical analysis as an assessment tool in a student compounding laboratory does carry some burdens for the course instructor and the school of pharmacy. ${ }^{6}$ The equipment and supplies for analyzing compounded preparations are costly. Furthermore, the analysis process is time intensive, especially with a large class size requiring auxiliary staff to carry out the analytical procedures in an expedient manner. However, the ability to demonstrate standards of practice firsthand to students based on evidence based data is worth the time and expense.

One limitation of each study was that only one suspension sample could be analyzed per student per time point. Each study covered a period of weeks, involved an inordinate amount of analysis time, and was conducted while other compounding exercises were being completed by the students. Almost all of the students' compounded preparations are analyzed in this laboratory and so considerations had to be given to equipment, resources, and time. However, there were many studies conducted in the entirety of this report, and several hundred students were involved. It is therefore felt that the data is valid even in light of this "one sample" limitation. It was evident that the intervention applied in the final hypothesis study regarding 


\section{American Journal of Pharmaceutical Education 2019; 83 (5) Article 6781.}

Table 6. HPLC AUC Ratio of Zonisamide/Internal Standard for Suspensions Compounded on Day 0 and Analyzed Week After Week in the Final Hypothesis Study

\begin{tabular}{lcccccccc}
\hline Days & $\mathbf{0}$ & $\mathbf{7}$ & $\mathbf{1 4}$ & $\mathbf{2 1}$ & $\mathbf{2 8}$ & $\mathbf{3 5}$ & $\mathbf{4 2}$ & $\mathbf{4 9}$ \\
\hline SS RT & 0.33 & 0.56 & 0.69 & 0.68 & 0.70 & 0.61 & 0.69 & 0.66 \\
MC RT & 0.26 & 0.64 & 0.68 & 0.72 & 0.71 & 0.69 & 0.71 & 0.71 \\
SS Ref & 0.59 & 0.67 & 0.61 & 0.67 & 0.60 & 0.55 & 0.64 & - \\
MC Ref & 0.68 & 0.74 & 0.67 & 0.70 & 0.72 & 0.72 & 0.72 & - \\
\hline
\end{tabular}

Abbreviations: $\mathrm{SS}=$ simple syrup, $\mathrm{MC}=$ methylcellulose, $\mathrm{Ref}=$ refrigeration, $\mathrm{RT}=$ room temperature

\# of replicates: $\mathrm{n}=1$

proper shaking and sample taking improved dosing accuracy. The impact on students actually seeing the results of the ongoing intervention was well worth all of the efforts.

\section{CONCLUSION}

The study began with a straightforward objective to teach students to determine the BUD of a compounded suspension. However, what the study ultimately demonstrated was to correctly dose a suspension requires explicit and clear, verbal instructions from a person who knows compounding sciences and understands the importance of properly shaking a suspension and correctly drawing a dose. Since the dosing results from a correct sampling technique, the students learned that correct sampling techniques are likewise necessary to accurately determine the true BUD of a compounded zonisamide suspension. By implication, the study showed a pharmacist's verbal intervention about correctly shaking and sampling a suspension is critical for patients to prepare accurate doses at home to maximize their therapeutic outcomes. Furthermore, a suspension must also be correctly shaken before a pharmacist dispenses a suspension to a patient, as reported to the Institute of Safe Medication Practices. ${ }^{7}$ Thus, the "shake well" auxiliary label associated with any suspension should create an urgency on the part of the pharmacist to ensure that both they and their patients shake the suspension properly before use.

\section{REFERENCES}

1. Abobo CV, Wei B, Liang D. Stability of zonisamide in extemporaneously compounded oral suspensions. Am J Health-Syst Pharm. 2009:66(12);1105-1109.

2. Almoazen H, Samsa AC, May CN. Why analytical testing is needed in pharmaceutical compounding. Am J Pharm Educ. 2010;74(2):Article 32.

3. Kadi A, Francioni-Proffitt D, Hindle M, Soine W. Evaluation of basic compounding skills of pharmacy students. Am J Pharm Educ. 2005;69(4):Article 69.

4. McGill JE, Holly DR. Integration of pharmacy practice and pharmaceutical analysis: quality assessment of laboratory performance. Am J Pharm Educ. 1996;60(4):370-374.

5. Pignato AP, Birnie CR. Analysis of compounded pharmaceutical products to teach the importance of quality in an applied pharmaceutical laboratory course. Am J Pharm Educ. 2014;78 (3):Article 61.

6. Roark AM, Anksorus HN, Shrewsbury RP. Long-term results of an analytical assessment of student compounded preparations. Am J Pharm Educ. 2014;78(9):Article 164.

7. Kelly K. Shake well before dispensing. Pharmacy Times. 2005; October 1, 2005-10-9921. 


\section{American Journal of Pharmaceutical Education 2019; 83 (5) Article 6781.}

Appendix 1. Master Formulation Record (Abbreviated)

Name: Zonisamide Suspension

Strength: $10 \mathrm{mg} / \mathrm{mL}$

Dosage Form: Suspension

Route of Administration: Oral

\begin{tabular}{|c|c|c|c|c|}
\hline Ingredient & Quantity & Physical Description & Solubility & Therapeutic Activity \\
\hline $\begin{array}{l}\text { Zonisamide } \\
\quad \text { Capsule, } 100 \mathrm{mg}\end{array}$ & 4 capsules & $\begin{array}{l}\text { orange and white capsules, } \\
\text { W990 on white half: } \\
\text { zonisamide is a white powder }\end{array}$ & $\begin{array}{l}\text { (active ingredient) } 0.8 \mathrm{mg} / \mathrm{mL} \text { in } \\
\text { water, } 0.5 \mathrm{mg} / \mathrm{mL} \text { in } 0.1 \mathrm{~N} \mathrm{HCl}\end{array}$ & $\begin{array}{l}\text { Anticonvulsant: } \\
\text { partial seizures in } \\
\text { adults with epilepsy }\end{array}$ \\
\hline Simple Syrup NF & qs $40 \mathrm{~mL}$ & $\begin{array}{l}\text { viscous, slightly yellow liquid: } \\
\text { sucrose is a monoclinic } \\
\text { sphenoidal crystal }\end{array}$ & $\begin{array}{l}\text { (active ingredient) } 1 \mathrm{~g} / 0.5 \mathrm{~mL} \text { water; } \\
\text { simple syrup is miscible with water }\end{array}$ & Vehicle \\
\hline OR & & & & \\
\hline $\begin{array}{l}0.5 \% \\
\quad \text { Methylcellulose } \\
1500 \mathrm{cps}\end{array}$ & qs $40 \mathrm{~mL}$ & $\begin{array}{l}\text { colorless, opaque low viscosity } \\
\text { gel: MC is white granules }\end{array}$ & $\begin{array}{l}\text { (active ingredient) soluble in cold } \\
\text { water; insoluble in hot water: } 0.5 \% \\
\text { MC is water miscible }\end{array}$ & Vehicle \\
\hline
\end{tabular}

Equipment Required:

mortar and pestle

$50 \mathrm{~mL}$ graduated cylinder

$2 \mathrm{oz}$. plastic prescription bottle, $22 \mathrm{~mm}$ oral syringe adapter

Method of Preparation:

1. Calibrate the 2 oz. bottle with water to $40 \mathrm{~mL}$.

2. Empty the contents of 4 zonisamide capsules into a mortar, and add a portion of the assigned vehicle. Triturate with a pestle.

3. Transfer the mortar contents into the $2 \mathrm{oz}$. bottle. Continue to add portions of the vehicle to the mortar to rinse the mortar until the $2 \mathrm{oz}$. bottle is brought to volume. Shake well.

4. Label the bottle with your name, date compounded, assigned student number, assigned vehicle, assigned storage temperature.

Description of Finished Preparation:

Preparation with Simple Syrup: Opaque suspension with slight off-white or yellowish color; moderate viscosity.

Preparation with methylcellulose: Opaque white suspension with moderate viscosity; bubbles present.

Quality Control Procedures:

\begin{tabular}{lllll}
\hline $\begin{array}{l}\text { Days after } \\
\text { Compounding }\end{array}$ & $\begin{array}{c}\text { Chemical Stability } \\
\text { Sample Drawn? }\end{array}$ & Color Change & Microbial Growth & Odor Present \\
\hline 0 & & & \\
7 & & & \\
14 & & & \\
21 & & & \\
28 & & & \\
\hline
\end{tabular}

1. For Chemical Stability Study: On the sample day, SHAKE the 2 oz. bottle well enough to suspend all of the capsule contents on the bottom. Carefully draw a $0.5 \mathrm{~mL}$ sample using a $1 \mathrm{~mL}$ oral syringe. Place the sample in a scintillation vial. Turn in for analysis with a vial label.

2. For Physical Stability Study: After completing the Chemical Stability Study, shake the 2 oz. bottle again, and pour about $10 \mathrm{~mL}$ of the product into the scintillation vial. Observe the suspension for the three stability parameters in the table above and report the data as yes/no. Return the $10 \mathrm{~mL}$ of suspension back to the $2 \mathrm{oz}$. bottle and shake well. Return the bottle to the assigned storage place. 


\section{American Journal of Pharmaceutical Education 2019; 83 (5) Article 6781.}

Packaging Container: Package in $2 \mathrm{oz}$. plastic prescription bottle with a $22 \mathrm{~mm}$ oral syringe adapter.

Storage Requirements: You will be assigned to either store your preparation at room temperature or at refrigerated temperature.

Beyond-Use Date Assignment: To be determined in the laboratory exercise.

Label Information:

Source of Recipe: Am J Health-Syst Pharm 66:1105-1109 (2009)

Literature 